\title{
Strand and Cable R\&D for Fast Cycled Magnets at CERN
}

\author{
L. Bottura, A. Bonasia, F. Borgnolutti, W. Gaertner, S. Le Naour, L. Oberli, G. Peiro, D. Richter, T. Salmi, \\ G. Sikler, and G. Willering
}

\begin{abstract}
Fast cycled superconducting magnets (FCM's) are an option of interest for the long-term consolidation and upgrade plan of the LHC accelerator complex. In the past two years we have conducted an $R \& D$ targeted at investigating the feasibility, operational issues and economical advantage of FCM's in the range of $2 \mathrm{~T}$ bore field, continuously cycled at $1 \mathrm{~Hz}$. In this paper we report the main results on the development of strands and cables suitable for this application, providing details on the strands tested and the cable manufacturing and performance.
\end{abstract}

Index Terms-AC loss, superconducting accelerator magnets, superconducting cables, superconducting filaments and wires.

\section{INTRODUCTION}

$\mathbf{E}$ NERGY efficient, superconducting, fast-cycled magnets (FCM's) would be a holy grail for accelerator applications including high energy physics, nuclear physics, or hadron therapy. Parallel and complementary to on-going development at FAIR [1] and INFN [2], we have launched at CERN an R\&D to explore the possibility to produce dipole fields in the range of $2 \mathrm{~T}$ at $1 \mathrm{~Hz}$ taking advantage of the superferric concept pursued for the FAIR SIS-100 accelerator. A sketch of the demonstration magnet that we are building is shown in Fig. 1. The main feature of the magnet is the use of a cryostated coil in a warm iron yoke, which should yield overall cryogenic loss below $5 \mathrm{~W} / \mathrm{m}$ of magnet [3]. This is a factor approximately 3 smaller than the present GSI SIS-100 design that we take as a benchmark [4].

The first, and core element of this R\&D is the production and qualification of a suitable strand and cable. In this paper we give a summary of the results of the cable production and characterization. We start by describing the strands used and their measured properties, we give details on the cable manufacturing, and we finally report the main results of the tests performed in the FReSCa test facility at CERN [5]. The objective is to provide a benchmark for the performance of the demonstration magnet.

\section{STRANDS}

\section{A. Strand Variants}

For our development we have used two different Nb-Ti strands with properties summarized in Table I. The first is an

Manuscript received August 02, 2010; accepted November 15, 2010. Date of publication February 14, 2011; date of current version May 27, 2011.

L. Bottura, A. Bonasia, F. Borgnolutti, S. Le Naour, L. Oberli, G. Peiro, D. Richter, and G. Willering are with CERN, Geneva, Switzerland (e-mail: Luca.Bottura@ cern.ch).

T. Salmi is with LBNL, Berkeley, CA, USA.

W. Gaertner and G. Sikler are with BNG, Wurzburg, Germany.

Color versions of one or more of the figures in this paper are available online at http://ieeexplore.ieee.org.

Digital Object Identifier 10.1109/TASC.2011.2105236

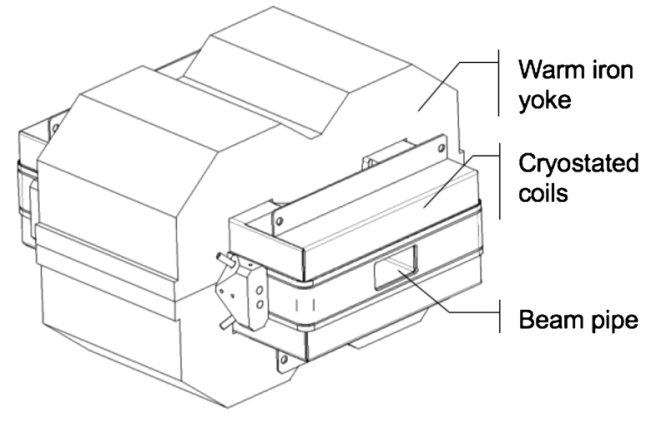

Fig. 1. 3-D view of the FCM demonstration magnet, showing the cryostated superconducting coil assembly installed in a warm iron.

TABLE I

MAIN CHARACTERISTICS OF THE STRANDS

\begin{tabular}{cccc}
\hline Strand & & 46B01428 & 46B14040 \\
\hline Diameter & $(\mathrm{mm})$ & 0.6 & 0.6 \\
Twist pitch & $(\mathrm{mm})$ & 6 & 10 \\
Cu:CuMn:NbTi & $(-)$ & $1.62: 0: 1$ & $2.39: 0.47: 1$ \\
RRR & $(-)$ & 58 & 110 \\
$J_{C}(5 \mathrm{~T}, 4.2 \mathrm{~K})$ & $\left(\mathrm{A} / \mathrm{mm}^{2}\right)$ & 2880 & $1875-2015$ \\
$n$ index & $(-)$ & $45-50$ & $10-25$ \\
\hline
\end{tabular}

LHC inner cable strand, drawn from 1.065 to $0.6 \mathrm{~mm}$ so to decrease the filament diameter from the nominal $7 \mu \mathrm{m}$ to an expected $4 \mu \mathrm{m}$. A cross section of this single-stacked strand is reported in Fig. 2, showing the 8900 round filaments regularly spaced. To be noted that this strand did not undergo adapted annealing steps during the additional drawing, which explains the relatively low RRR (approximately 60) when compared to the standard of the LHC production (150 and above). The measured $J_{C}$ of $2880 \mathrm{~A} / \mathrm{mm}^{2}$ at $5 \mathrm{~T}$ and $4.2 \mathrm{~K}$ (referred to the $\mathrm{Nb}-\mathrm{Ti}$ cross section), and the large $n$ index (45 to 50), are in close agreement with the performance of the same strand at the nominal diameter, thus indicating no significant degradation during the drawing to the reduced diameter.

The second strand type used for cabling is a double-stacked $R \& D$ billet that we manufactured jointly with industry using a mixed $\mathrm{Cu}$ and $\mathrm{Cu}-\mathrm{Mn}$ matrix. The strand, shown in Fig. 3, has $14040 \mathrm{NbTi}$ filaments with a nominal diameter of $2.6 \mu \mathrm{m}$, and a nominal spacing of $0.5 \mu \mathrm{m}$.

At such reduced spacing the filaments may couple electromagnetically [6], hence the choice of the alloyed matrix to take full benefit of the small geometric filament. The sub-elements consist of $78 \mathrm{Nb}$-Ti filaments in a $\mathrm{Cu}-0.58$ at\% $\mathrm{Mn}$ matrix, drawn 

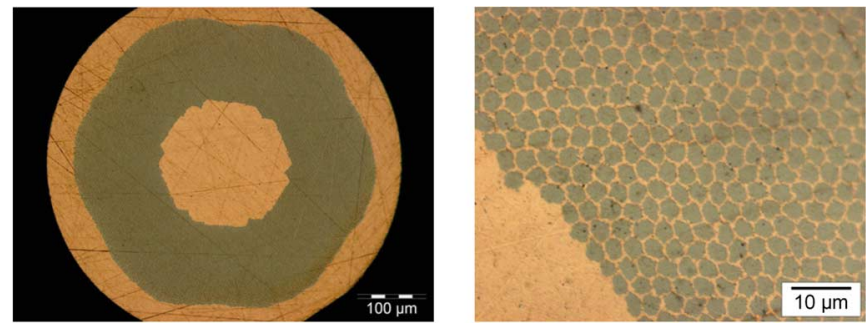

Fig. 2. Cross section of the Copper matrix, Nb-Ti strand (46B01428), and detail of the filaments in the copper matrix.
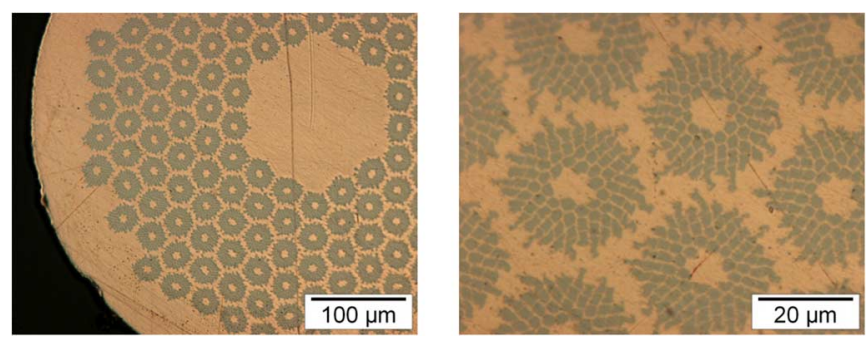

Fig. 3. Cross section of the Mixed (Cu/Cu-Mn), Nb-Ti strand (46B14040), and detail of the filament bundles.

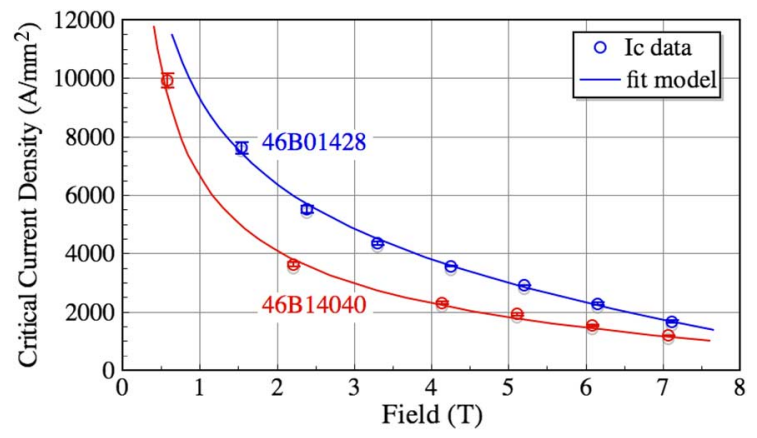

Fig. 4. Critical current density at $4.2 \mathrm{~K}$, as a function of magnetic field.

to hexagonal shape for re-stacking. The final billet contains 180 such sub-elements around an assembly of $19 \mathrm{Cu}$ hexagons, in a $\mathrm{Cu}$ canister, extruded and drawn to the final diameter. As can be seen from the micrographs in Fig. 3, the difference in material properties caused excessive deformation in the outer perimeter of the sub-element during the extrusion step, after restacking. In spite of the large filament deformation, this wire reached a best measured $J_{C}$ of $2015 \mathrm{~A} / \mathrm{mm}^{2}$ at $5 \mathrm{~T}$ and $4.2 \mathrm{~K}$ (referred to the $\mathrm{Nb}-\mathrm{Ti}$ cross section), albeit with a relatively low $n$ (in the range of 10 to 25) and large scattering on $J_{C}$ samples in the billet $( \pm 3.5 \%)$.

\section{B. Strand Tests}

The critical current of strand samples from the same piece lengths used for cabling was measured in the range of 0 to 7 $\mathrm{T}$ applied field, at $4.2 \mathrm{~K}$ and for currents below $1 \mathrm{kA}$. The result of the measurement is reported in Fig. 4, together with the fit defined in [7], with parameters adjusted to each of the two strands. Note that because of the small diameter and the large critical current at low field, it was important to apply a self-field

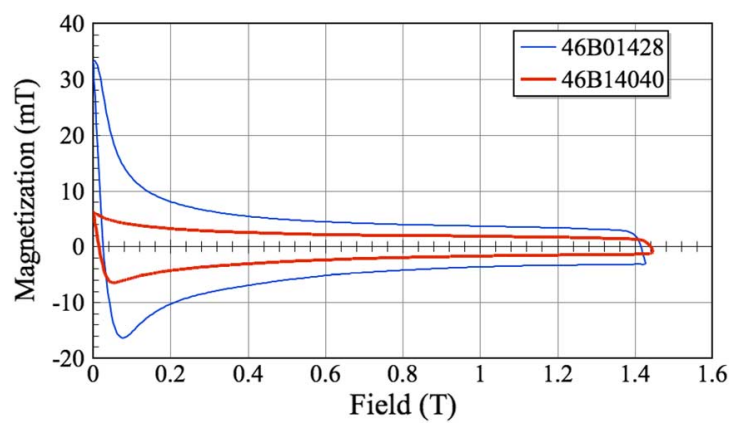

Fig. 5. Volume magnetization of the two strand variants, at $4.3 \mathrm{~K}$.

TABLE II

HYSTERESIS LOSS FOR TRAPEZOIDAL CYCLES

\begin{tabular}{cccccc}
\hline \multicolumn{2}{c}{ Strand } & \multicolumn{2}{c}{$46 \mathrm{~B} 01428$} & \multicolumn{2}{c}{$46 \mathrm{~B} 14040$} \\
\hline $\mathrm{B}_{\max }$ & $(\mathrm{T})$ & 0.8 & 1.45 & 0.8 & 1.45 \\
$\mathrm{Q}_{\mathrm{H}}$ & $\left(\mathrm{mJ} / \mathrm{cm}^{3}\right)$ & 10.2 & 13.3 & 3.5 & 5.2 \\
\hline
\end{tabular}

correction to the data, based on the peak field in the multi-filamentary region. At zero applied field only the mixed matrix strand (46B14040), with $I_{C}<1 \mathrm{kA}$ could be measured.

We further measured the magnetization $M$ and hysteresis loss $Q_{H}$ per unit strand volume using the set-up described in [8]. The two strand types were tested under trapezoidal field sweeps $\left(0-B_{\max }-0\right)$ in the range of $B_{\max }$ from 0 to $1.45 \mathrm{~T}$. The measured magnetization for the $1.45 \mathrm{~T}$ peak field cycle is reported in Fig. 5, while AC loss data, obtained from the integral of the magnetization curves, are given in Table II. At first sight, the mixed matrix strand 46B14040 shows much reduced magnetization, no coupling, and smaller hysteresis loss per unit strand volume when compared to the copper matrix strand 46B01428. In reality, most of the reduction can be explained by the different volume fractions and the lower $J_{C}$ of strand 46B14040. Indeed, an evaluation of the effective filament diameter from the width of the magnetization loop at $1 \mathrm{~T}$ yields approximately $4 \mu \mathrm{m}$ for both strands, which is most likely due to the fact that a large fraction of filaments in the mixed matrix strand is strongly deformed and may be coupled.

\section{CABLES}

\section{A. Cable Design}

We have chosen for this application an internally cooled cable where the strands are twisted around a cooling pipe and held in place by a tight wire wrap. This is the configuration originally developed for the Nuclotron [9] and now planned to be used for the manufacturing of the SIS- 100 dipoles at FAIR [1]. In our case, the design point is for a maximum current of $6 \mathrm{kA}$ at a peak background field of $2 \mathrm{~T}$ and $4.3 \mathrm{~K}$ operating temperature.

The internal pipe is a seamless tube of CuNi30Mn1 alloy with $5 \mathrm{~mm}$ ID and $6 \mathrm{~mm}$ OD. The tube is in half-hardened state $\left(\sigma_{\text {yield }} \approx 120 \mathrm{MPa}, \sigma_{\text {ultimate }}>370 \mathrm{MPa}\right.$ ), which is the best compromise between the required mechanical strength and the need for winding in a coil. 
The number of strands that can be cabled has an upper limit given by the available space on the developed outer circumference of the pipe. The trivial upper limit is obtained dividing the developed circumference $\pi\left(O D_{\text {pipe }}+D_{\text {strand }}\right)$ by the strand diameter $D_{\text {strand }}$, which in our case yields a maximum of 34 strands. In reality, because of the twist pitch, the strands occupy a larger fraction of the circumference. Indicating the $\mathrm{ca}$ bling angle with $\theta$, we expect to be able to cable not more than $\pi \cos (\theta)\left(1+O D_{\text {pipe }} / D_{\text {strand }}\right)$ strands around the pipe. Taking a cabling angle in the common range of 15 degrees, we obtain an upper limit of 33 strands, and a remaining tolerance of 0.2 $\mathrm{mm}$ on the circumference. As discussed later, this upper limit cannot be achieved in practice without adjustment.

The main function of the outer wrap is to guarantee a good contact between the strands and the pipe under all working conditions. For this reason we have taken a Ni-Cr8020 wire of 0.3 $\mathrm{mm}$ diameter, which has sufficient strength to apply a high centering force $\left(\sigma_{\text {ultimate }} \approx 800 \mathrm{MPa}\right)$. We have set the wrap force at approximately $10 \mathrm{~N}$, and the wrap pitch at $0.5 \mathrm{~mm}$, resulting in a design force per unit length of $20 \mathrm{kN} / \mathrm{m}$. This has a large margin, up to two orders of magnitude with respect to the electromagnetic force experienced by a strand in operating conditions.

\section{B. Cable Manufacturing}

The cable is manufactured feeding the straightened central $\mathrm{Cu}-\mathrm{Ni}$ pipe through the barrels in the cabling machine, and the pipe is used as cabling mandrel. A cabling eye was installed close to the merging point of the strands on the pipe, with tight tolerances $(<0.1 \mathrm{~mm})$ with respect to the outer dimension of the cable before wrapping. This makes the initial threading of the strands delicate and time consuming, but is necessary to avoid strand overlap during cabling. The $\mathrm{Ni}-\mathrm{Cr}$ wrap is applied right after the cabling eye using a hollow spindle turning at high speed. Indeed, the wrapping stage gives the upper limit to the cable manufacturing speed, typically in the range of $0.3 \mathrm{~m} / \mathrm{min}$ for a spindle rotating at $600 \mathrm{rpm}$.

Tests were performed at the beginning of the production using $\mathrm{Cu}$ wire leaders of the same diameter as the superconducting strands, to find the cabling parameters that gave the best results. We have obtained stable cable configuration, with no overlaps or signs of shaving at the cabling eye, cabling 32 strands with a twist pitch of $70 \mathrm{~mm}(\cos (\theta)=0.959)$, or 33 strands with a twist pitch of $86 \mathrm{~mm}(\cos (\theta)=0972)$. This implies that it is possible to fill the theoretical developed circumference only up to a filling factor in the range of 97 to $98 \%$. In practice, it is a good rule-of-thumb to manufacture the cable with one strand less than the number that would result in the ideal filling of the pipe circumference.

We finally proceeded to manufacture 10 unit lengths $(80 \mathrm{~m})$ of two cable variants (FCM001 and FCM002) based on the above parameters, whose main characteristics are reported in Table III. The reason for this choice was to use the strand type with highest $I_{C}$ in the cable with least strands, so to balance somewhat the difference between strand type. It is worth mentioning that the use of superconducting strands did not give noticeable changes with respect to the $\mathrm{Cu}$ wires in the dummy lengths, and that
TABLE III

Main CHARACTERISTICS OF THE CABLE VARIANTS MANUfACTURED

\begin{tabular}{cccc}
\hline Cable & & FCM001 & FCM002 \\
\hline Strand & & 46B01428 & $46 \mathrm{~B} 14040$ \\
Strands & $(-)$ & 32 & 33 \\
Twist pitch & $(\mathrm{mm})$ & 69.5 & 85.9 \\
Outer diameter & $(\mathrm{mm})$ & \multicolumn{3}{c}{7.74} \\
\hline
\end{tabular}

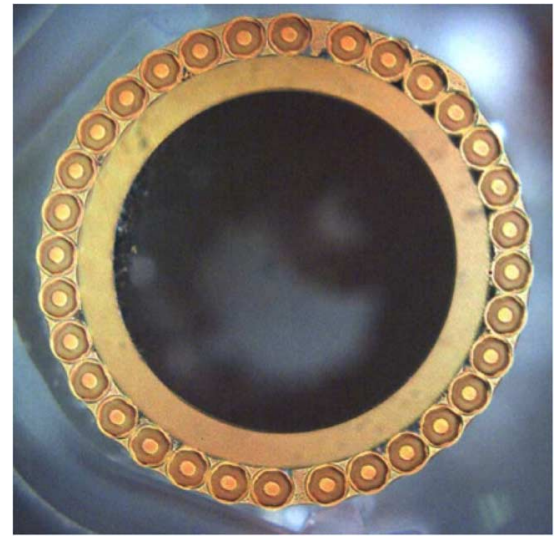

Fig. 6. Cross section of finished cable, showing the central pipe and the cabled strands, with the outer $\mathrm{N}-\mathrm{Cr}$ wrap removed. The solder between strands was used during sample preparation, and is not present in the finished cable.

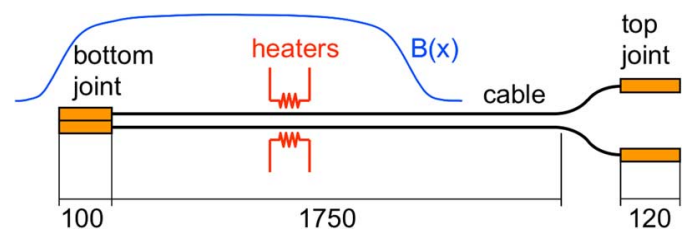

Fig. 7. Schematic of a FReSCa cable sample, lengths are in $\mathrm{mm}$. The sample is inserted vertically in the test station.

the strand electrical characteristics $\left(J_{C}\right.$ and $\left.R R R\right)$ were not affected by the cabling, as verified on extracted strands. A sample cross section of finished cable is shown in Fig. 6.

\section{CABle Tests}

\section{A. Experimental}

Two hairpin samples were prepared from the two cable variants, and tested at $4.2 \mathrm{~K}$ in the FReSCa test station [5]. A schematic view of a sample is show in Fig. 7. It consists of two legs of the same cable, approximately $2 \mathrm{~m}$ long, mounted on a sample holder specifically developed for this task. At the bottom of the sample the cables are soldered in a copper shoe, which is then fastened to form a demountable electrical joint. The joints at the top of the sample are done using the same principle, and they are clamped to the current terminals of the test insert. Heaters are mounted on each cable to initiate quenches, covering a $50 \mathrm{~mm}$ length in the center of the uniform field region of the background magnet. Each cable is instrumented with 10 voltage taps distributed along the whole cable length. They are used to localize quenches, to measure the voltage 


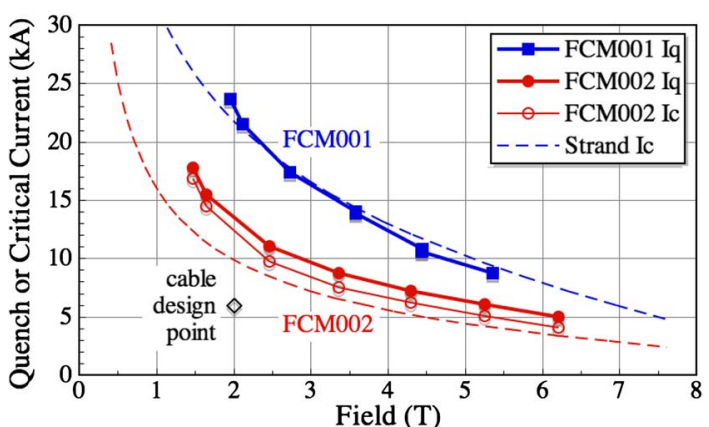

Fig. 8. Quench and critical current versus magnetic field (corrected for self field) in the two cable samples at $4.3 \mathrm{~K}$. The current ramp rate for the measurement is $1 \mathrm{kA} / \mathrm{s}$. Also reported the expected critical current from strand data, as well as the cable design point.

development after a resistive transition, and to estimate the normal propagation zone velocity.

Once inserted in the test cryostat, the sample was cooled down to liquid helium temperature, approximately $4.3 \mathrm{~K}$. The bath-cooling conditions for the cable sample are not the same as those foreseen in the FCM magnet (the cable is supposed to be internally cooled by a forced flow supercritical helium) but they are appropriate to qualify the cable and to make a direct comparison of the two cable variants. Various tests were performed during a campaign: quench current versus background field, current ramp rate dependence, joint resistance, and quench initiation and propagation.

\section{B. Quench and Critical Current Measurements}

The quench current $I_{Q}$ of both cable samples was measured at constant background field, $4.3 \mathrm{~K}$ and $1 \mathrm{kA} / \mathrm{s}$ ramp-rate. The measured values are reported in Fig. 8, plotted against the total field (background and self-field). The first sample trained significantly before reaching stable quench current. We believe that the reason for the training is the use of polyethylene spacers in the sample holder, which did not have sufficient rigidity to withstand the electromagnetic force. Replacing the spacer with more rigid, fiber-glass loaded, epoxy pieces eliminated the training altogether.

We first remark that although there is a clear difference in their current carrying capability, both cable variants have ample margin with respect to the cable design point $(6 \mathrm{kA}$ at $2 \mathrm{~T})$, also indicated in the plot. Furthermore, once the two data sets are plotted using the same self-field correction (peak-field), the cable performance is either very close (FCM001) or even exceeds (FCM002) the expected critical current from the strand data. This essentially qualifies the cable design and manufacturing process.

In the case of cable FCM002, outperforming the single strand by $25 \%$, we analysed the resistive transitions to make the comparison more meaningful. Defining a critical current $I_{C}$ with the same criterion used for the strand, i.e., $10^{-14} \Omega \mathrm{m}$ resistivity, we see in Fig. 8 that the discrepancy between cable and strand performance decreases to 10 to $15 \%$, which is more acceptable. Note that the same type of analysis does not affect the results of cable FCM001 as in this case the $n$-index of the transition is large (30 to 40). Indeed, as remarked earlier, the strand used for cable FCM002 already had a low $n$-index (10 to 20). It is

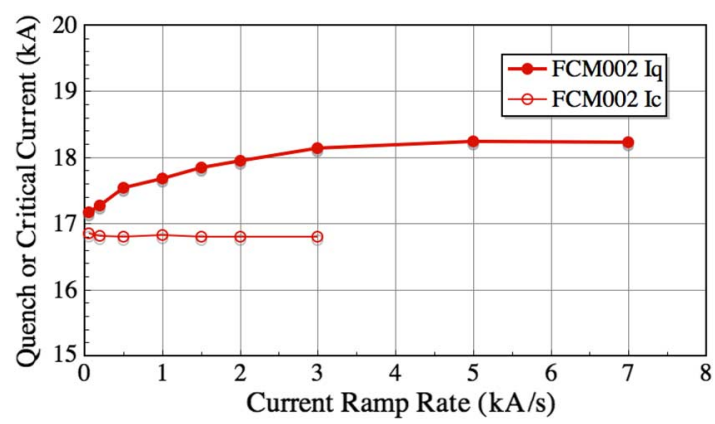

Fig. 9. Quench and critical current versus current ramp-rate, measured for the FCM002 cable sample, in zero background field and at $4.3 \mathrm{~K}$.

conceivable that under this condition the current redistributes during a resistive transition, thus explaining the excess current carrying capability. This also implies that the self-field correction assumed is probably excessive, i.e., the effective field seen by the cable at a quench is not the peak field. We hence attribute the remaining discrepancy between strand and cable $I_{C}$ to the combination of these two effects that are hard to quantify, but inessential for the qualification of the cable for the expected use in a magnet.

\section{Ramp-Rate Dependence}

The projected application for the FCM cable includes continuous cycling at relatively high ramp-rate, i.e., $1.5 \mathrm{~T} / \mathrm{s}$ field ramp-rate $\mathrm{dB} / \mathrm{dt}$ and $6 \mathrm{kA} / \mathrm{s}$ current ramp-rate $\mathrm{dI} / \mathrm{dt}$. We hence tested the dependence of the quench current on $\mathrm{d} / / \mathrm{dt}$. For this test we set the background field to $0 \mathrm{~T}$ (the FReSCa dipole is not adapted for operation at large $\mathrm{dB} / \mathrm{dt}$ ) and performed ramps to quench with $\mathrm{dI} / \mathrm{dt}$ varying from $50 \mathrm{~A} / \mathrm{s}$ to $7 \mathrm{kA} / \mathrm{s}$. A sample plot of $I_{Q}$ and $I_{C}$ versus $d I / d t$ for the cable FCM002 is shown in Fig. 9. The slight increase of $I_{Q}$ in this range is due to the dynamic nature of the transition, meaning that the heating above the voltage criterion contributes to the build-up towards runaway conditions. Faster ramps produce less heat, and result in a higher runaway current $I_{Q}$. The critical current $I_{C}$, on the other hand, does not show any significant dependence. Note that the peak value of the self-field generated by the cable sample is approximately $80 \mathrm{mT} / \mathrm{kA}$, and $d I / d t$ of $7 \mathrm{kA} / \mathrm{s}$ corresponds to $d B / d t$ of $0.5 \mathrm{~T} / \mathrm{s}$, relevant for the projected operating conditions in the magnet.

\section{CONCLUSION}

We have successfully manufactured cables for fast cycled accelerator magnet applications, and more specifically for the FCM magnet being built at CERN as a technology demonstration of a low-loss superferric dipole. Both strands and cables are coming from industrial productions, suitable for long lengths and large quantities. An analysis of the manufacturing parameters, especially for the cables, has resulted in practical recipes useful to produce variants or new designs.

The cable variants produced were qualified by short sample tests. They demonstrated no degradation versus the strand performance, and ample margin with respect to the design conditions. We plan to pursue our R\&D with complementary characterizations (a coupling loss measurement is planned to provide a benchmark for the loss properties). 
The available length of cable (approximately $800 \mathrm{~m}$ in 10 unit lengths) will be used to produce magnet poles to be tested in the FCM demonstration magnet, with results expected in mid 2011.

\section{REFERENCES}

[1] P. J. Spiller et al., "Design status of the FAIR synchrotrons SIS100 and SIS300 and the high energy beam transport system," in Proceedings of EPAC08, Genoa, Italy, Jun. 2008, pp. 298-300, 2008.

[2] P. Fabbricatore et al., "Development of a curved fast ramped dipole for FAIR SIS300," IEEE Trans. Appl. Supercond., vol. 18, no. 2, pp. 232-235, 2008.

[3] L. Bottura et al., W. Scandale and F. Zimmermann, Eds., "The fast cycled superconducting magnets (FCM) R\&D Program at CERN," in Proceedings of $\mathrm{HHH}-2008$, Nov. 2008 [Online]. Available: http://carehhh.web.cern.ch/CARE-HHH/HHH-2008/proceedings_hhh2008.htm.
[4] A. Kovalenko et al., "New results on minimizing AC power losses in a fast cycling 2 T superferric dipole with a cold yoke," IEEE Trans. Appl. Supercond., vol. 16, no. 2, pp. 338-341, 2006.

[5] A. P. Verweij et al., "1.9 K Test facility for the reception of the superconducting cables for the LHC,' IEEE Trans. Appl. Supercond., vol. 9, no. 2, pp. 153-156, 1999.

[6] A. K. Ghosh and W. B. Sampson, "The effect of magnetic impurities and barriers on the magnetization and critical current of fine filaments NbTi composites," IEEE Trans. Mag., vol. 24, no. 2, pp. 1145-1148, 1988.

[7] L. Bottura, "A Practical fit for the critical surface pf NbTi," IEEE Trans. Appl. Sup., vol. 10, no. 1, pp. 1054-1057, 2000.

[8] S. Le Naour et al., "Magnetization measurements on LHC superconducting strands," IEEE Trans. Appl. Sup., vol. 9, no. 2, pp. 1763-1766, 1999.

[9] A. D. Kovalenko, "Status of the nuclotron," in Proceedings of EPAC94,, London, England, Jun. 1994, pp. 161-164, 1995. 